\title{
Antibacterial activities of the methanol extract, fractions and compounds from Elaeophorbia drupifera (Thonn.) Stapf. (Euphorbiaceae)
}

Igor K. Voukeng ${ }^{1}$, Blaise K. Nganou², Louis P. Sandjo ${ }^{3}$, Ilhami Celik', Veronique P. Beng ${ }^{5}$, Pierre Tane ${ }^{2}$ and Victor Kuete ${ }^{1 *}$

\begin{abstract}
Background: Elaeophorbia drupifera (Thonn.) Stapf. (Euphorbiaceae) is used in Cameroonian folk medicine to treat several ailments including bacterial-related diseases such as skin infections. In this study, the methanol extract from the leaves (EDL), fractions (EDLa-d), sub-fractions EDLc1-7 and EDLC31-35 as well as isolated compounds were tested for their antimicrobial activities against a panel of Gram-negative and Gram-positive bacteria including multidrug resistant (MDR) phenotypes.

Methods: The broth microdilution method was used to determine the minimal inhibitory concentration (MIC) and minimal bactericidal concentration (MBC) of the above samples; column chromatography was used for the fractionation and purification of the leaves extract whilst the chemical structures of compounds were determined using spectroscopic techniques.

Results: Phytochemical investigation lead to the isolation of a mixture (1:3) of stigmasterol and $\beta$-sitosterol $(1+2)$, euphol (3), sitosterol-O- $\beta$ - - -xylopyranoside (4), 3,3', $4^{\prime}$-tri-O-methylellagic acid (5), a mixture (1:1) of afzelin and quercetin-3-O- $\beta$-D-xylopyranoside (6 + 7), 3,3',4'-tri-O-methylellagic acid 4-O- $\beta$-D-glucopyranoside (8), ellagic acid-4-O$\beta$-xylopyranoside-3,3',4'-trimethyl ether (9) from EDLc. Crude extract and fractions displayed selective activities with MIC values ranged from 32 to $1024 \mu \mathrm{g} / \mathrm{mL}$ for EDL against 84.9\% of the 33 tested bacteria, $93.9 \%$ for EDLc, 69.7\% for EDLb, 33.4\% for EDLa and $0.03 \%$ for EDLd. MIC values ranged from 16 to $1024 \mu \mathrm{g} / \mathrm{mL}$ were obtained with EDLC3 and EDLC4 on all tested bacteria meanwhile other sub-fractions displayed selective activities. MIC value of 32 $\mu \mathrm{g} / \mathrm{mL}$ was obtained with fractions EDLa against Escherichia coli AG100, EDLc against Enterobacer aerogenes ATCC13048 and EA298. For sub-fractions obtained from EDLC, the lowest MIC value of $16 \mu \mathrm{g} / \mathrm{mL}$ was recorded with EDLC3 against Staphylococcus aureus MRSA11. A corresponding value of $8 \mu \mathrm{g} / \mathrm{mL}$ against Providencia stuartii NAE16 was recorded with EDLC33 obtained from further fractionation of EDLC3. EDLC3 had MIC values below $100 \mu \mathrm{g} / \mathrm{mL}$ against all tested bacteria. Compound 5 as well as the mixture (1:1) of 6 and 7 inhibited the growth of all the tested bacteria with MICs ranged from 64 to $256 \mu \mathrm{g} / \mathrm{mL}$.

Conclusion: Elaeophorbia drupifera is a potential source of phytomedicine to tackle MDR bacteria. Sub-fraction EDLC3 was more active than all isolated compounds and deserves further investigations to develop natural drug to combat Gram-negative, Gram-positive bacteria and otherwise MDR phenotypes.
\end{abstract}

Keywords: Antibacterial, Crude extract, Compounds, Elaeophorbia drupifera, Fractions, Multi-drug resistance

\footnotetext{
* Correspondence: kuetevictor@yahoo.fr

1 Department of Biochemistry, Faculty of Science, University of Dschang, P.O.

Box 67, Dschang, Cameroon

Full list of author information is available at the end of the article
} 


\section{Background}

Despite the progress in antibiotherapy, the fight against bacterial infections still constitute a major concern worldwide. The antibacterial fight is seriously challenged by the development of of multi-drug resistant (MDR) phenotypes [1]. Scientists should take into account the resistance issue when investigating natural products for their antimicrobial potential. In regards of the biodiversity of plant kingdom, evidenced-based botanicals appear to be undeniable source of medicine to fight bacterial resistance [2, 3]. Recently, several bioactive plants against MDR bacteria were reported. Some of these include Erythrina sigmoidea [4], Cinnamomum zeylanicum, Aframomum citratum, Paullinia pinnata [5, 6], Allanblackia gabonensis, Combretum molle [7] and Harungana madagascariensis [8]. In our continous search of antibacterials from plants used traditionnally to manage microbial infections, we targeted Elaeophorbia drupifera (Thonn.) Stapf. (Euphorbiaceae). The plant is used in traditional medicine to treat skin infections, Guinea worm [9] as well as hypertension and diabetes [10]. Leaves extract was reported to moderately inhibit HIV-1 and HIV-2 proviral DNA copying [11], and to have relaxant effect on vascular smooth muscle on rats [12]. The leaves methanol extract of this plant also showed good cytotoxic activity towards leukemia CCRF-CEM and MDAMB231 breast cancer cell lines [13]. Previous phytochemical investigations of the plant led to the isolation of triterpenoids and steroids [14, 15]. In the present study, the bioguided fractionation was undertaken for depth investigation of the antibacterial activity of methanolic extract of Elaeophorbia drupifera leaves'.

\section{Methods}

\section{General procedure}

Mass spectral data [Electrospray ionization mass spectrometry (ESI-MS)] were measured on a Waters Synapt HDMS spectrometer. NMR Spectra were recorded with an Agillent spectrometer at $400 \mathrm{MHz}$. Chemical shifts $(\delta)$ were quoted in parts per million (ppm) from the internal standard tetramethylsilane (TMS). Deuterated dimethyl sulfoxide (DMSO- $d_{6}$ ), was used as solvent for the NMR experiments. Column chromatography was performed on silica gel Merck $60 \mathrm{~F}_{254}[(0.2-0.5 \mathrm{~mm})$ and (0.2-0.063 mm)] 70-230 and 230-400 mesh (Darmstadt, Germany). Pre-coated silica gel $60 \mathrm{~F}_{254}$ thin layer chromatography (TLC) plates (Merck, Germany) were used for monitoring fractions and spots were detected with UV light (254 and $365 \mathrm{~nm}$ ) and then sprayed with 50\% sulphuric acid $\left(\mathrm{H}_{2} \mathrm{SO}_{4}\right)$ followed by heating to $100{ }^{\circ} \mathrm{C}$.

\section{Plant material and extraction}

The leaves of Elaeophorbia drupifera were collected in Dschang, West Region of Cameroon $\left(5^{\circ} 27^{\prime} \mathrm{N} 10^{\circ} 04^{\prime} \mathrm{E}\right)$ in
January 2014. The plant was identified at the National Herbarium (Yaoundé, Cameroon) where a voucher specimen was deposited under the reference number $57644 /$ HNC [roots, leaves, bark]. The powder leaves of E. drupifera (1300 g) was soaked in methanol (MeoH; $5 \mathrm{~L})$ for $48 \mathrm{~h}$. After filtration and removal of the solvent using a rotary evaporator under reduced pressure, $233 \mathrm{~g}$ of crude extract (EDL) was obtained.

\section{Isolation and purification of bioactive compounds from the leaves extract of $E$. drupifera}

Part of crude extract (213 g) was dissolved in methanolwater $(9: 1 \mathrm{v} / \mathrm{v})$, followed by a liquid-liquid fractionation using $n$-hexane (Hex), dichloromethane $\left(\mathrm{CH}_{2} \mathrm{Cl}_{2}\right)$, and ethyl acetate (EtOAc) respectively. This afforded four fractions: $65 \mathrm{~g}$ EDLa (Hex), $48 \mathrm{~g}$ EDLb $\left(\mathrm{CH}_{2} \mathrm{Cl}_{2}\right), 91 \mathrm{~g}$ EDLc (EtOAc), and $12 \mathrm{~g}$ residual fraction (EDLd). Of these fractions, EDLc shows the best antibacterial activity and was therefore subjected to further purification processes.

Fraction EDLc (85 g) was subjected to column chromatography $(\mathrm{CC})$ over silica gel and eluted with increasing gradient of $n$-Hex-EtoAc and EtOAc - MeoH solvent mixtures. 167 Fractions of $300 \mathrm{~mL}$ each were collected; based on TLC results, they were combined into seven new sub- fractions tagged EDLc1 (7.5 g); EDLc2 (3.4 g); EDLc3 (8.5 g); EDLc4 (18.6 g); EDLc5 (17.3 g); EDLc6 (16.1 g) and EDLc7 (12.9 g). EDLc1 and EDLc7 displayed poor antibacterial effects and were not further investigated. EDLc2 (3.2 g) was subjected to CC over silica gel eluting with mixture of $n-\mathrm{Hex}-\mathrm{CH}_{2} \mathrm{Cl}_{2}$ mixtures of increasing polarity. 69 new sub-frs of 100 $\mathrm{mL}$ each were collected and combined into 4 other new sub-fractions; they were labelled EDLc21 (0.3 g); EDLc22 (1.2 g); EDLc23 (0.25 g) and EDLc24 (0.5 g). Sub-fractions 18-30 (EDLc22) was further purified by silica gel column chromatography eluting with n-hexane-Acetone (95:5) isocratic, to afford an amorphous solid identified and mixture (1:3) of stigmasterol and $\beta$-Sitosterol $(\mathbf{1}+\mathbf{2}$; $18 \mathrm{mg}$ ) and another white powder identified as euphol (3; $22 \mathrm{mg})$.

EDLc3 (7.5 g) was subjected to silica gel CC eluting with mixture solvents of $n$-Hex-EtOAc of increasing polarity. $141 \mathrm{sub}$ - fractions of $100 \mathrm{~mL}$ of each were collected and pooled into 5 sub-fractions: EDLc31 (0.6 g); EDLc32 (1.3 g); EDLc33 (1.9 g); EDLc34 (1.4 g); EDLc35 $(0.9 \mathrm{~g})$. The sub-fraction EDLc33 was subjected to further sephadex LH2O purification with $n-\mathrm{Hex}-\mathrm{CH}_{2} \mathrm{Cl}_{2}-\mathrm{MeOH}$ (9:2:0.5) mixture. Two new sub-fractions named EDL331 $(0.4 \mathrm{~g})$ and EDL332 (0.9 g) were obtained. EDL332 was further subjected to another silica gel CC eluting with dichloromethane- acetone $(98: 2)$ isocratic system to afford a white powder identified sitosterol $3-O-\beta-\mathrm{D}$-xylopyranoside (4; $405.2 \mathrm{mg}$ ) and a brownish powder identified 3,3',4'tri- $O$-methylellagic acid $(5 ; 8 \mathrm{mg})$. 
EDLc4 (18.5 g) was subjected to CC over silica gel eluting with $n$-Hex-acetone mixtures and $\mathrm{MeOH}$ of increasing polarity. 108 sub-fractions of $100 \mathrm{~mL}$ each were collected and regrouped based on their TLC profile into 4. sub-fractions named EDLc41 (3.8 g), EDLc42 (4.1 g), EDLc43 (3.6 g) and EDLc44 (2.8 g). Compound 5 (17 mg) was obtained in sub-fractions (EDLc41 - 44).

Sub-fraction EDLc5 (17.2 g) was subjected to silica gel $\mathrm{CC}$ eluting with a gradient of $\mathrm{CH}_{2} \mathrm{Cl}_{2}$-EtOAc and EtOAc-MeOH of increasing polarity, and was collected in 143 new sub-fractions of $100 \mathrm{~mL}$ each were collected. They were further pooled upon TLC analysis into five sub-fraction named EDLc51 (3.6 g), EDLc52 (2.2 g), $\operatorname{EDLc53}(2.4 \mathrm{~g}), \operatorname{EDLc} 54(1.9 \mathrm{~g})$ and $\operatorname{EDLc} 55$ (3 g). EDLc53 and EDLc54 were further subjected to Sephadex LH20 CC eluting with $n$-Hex- $\mathrm{CH}_{2} \mathrm{Cl}_{2}-\mathrm{MeOH}$ (7:4:0.5) mixture to afford a yellowish gum identified a mixture (1:1) of 3-O-rhamnopyranosyl kaemferol and 3-Oxylopyranosyl quercetin $(6+7 ; 18 \mathrm{mg})$.

Sub-fraction EDLc6 (16 g) was subjected to silica gel $\mathrm{CC}$ eluting with mixture of $\mathrm{CH}_{2} \mathrm{Cl}_{2}$-acetone and acetone-MeOH of increasing polarity. 119 new subfractions of $100 \mathrm{~mL}$ of were collected and combined based on their TLC profiles into 4 sub-fractions: EDLc61 (3.3 g), EDLc62 (2.4 g), EDLc63 (1.2 g) and EDLc64 (4.6 g). EDLc62 afforded a white powder identified as $3,3^{\prime}, 4^{\prime}$-tri- $O$-méthylelleargic acid $4-O-\beta^{-}{ }^{-}$ glucopyranoside $(\mathbf{8} ; 15.3 \mathrm{mg})$ and another white powder, ellagic acid-4-O- $\beta$-xylopyranoside- $3,3^{\prime}, 4^{\prime}$-trimethyl ether (9; $30 \mathrm{mg}$ ).

\section{Antimicrobial assays}

\section{Chemicals for antimicrobial assay}

The reference antibiotic (RA) used against bacteria was chloramphenicol $\geq 98 \%$ (Sigma-Aldrich, St. Quentin Fallavier, France) meanwhile the bacterial growth indicator was $p$-iodonitrotetrazolium chloride $\geq 97 \%$ (INT, SigmaAldrich).

\section{Microbial strains and culture media}

A panel of 33 bacteria belonging to Gram-negative and Gram-positive bacteria were investigated in this work. They included sensitive and resistant strains of Escherichia coli, Enterobacter aerogenes, Enterobacter cloacae, Klebsiella pneumoniae, Pseudomonas aeruginosa and Providencia stuartii (Gram-negative bacteria) as well as Staplylococcus aureus (Gram-positive bacteria). They were obtained clinically and from the American Type Culture Collection (ATCC). Their bacterial features were previously reported (Additional file 1: Table S1) [6]. Mueller Hinton Agar (Sigma) was used to activate the microorganisms whilst Mueller Hinton broth (MHB; Sigma) was used for antibacterial assays [16].

\section{INT colorimetric assay for MIC and MBC determinations}

The determinations MIC and MBC on the tested bacteria were monitored by the rapid INT colorimetric assay according to described methods [17] with some modifications [18, 19]. The test samples and RA were dissolved in dimethylsulfoxide (DMSO)/MHB. The final concentration of DMSO was lower than $2.5 \%$ and does not affect the microbial growth. The solution obtained was then added to $\mathrm{MHB}$, and serially diluted two fold (in a 96- wells microplate). The bacterial concentration was $1.5 \times 10^{6} \mathrm{CFU} / \mathrm{mL}$. The plates were incubated at $37{ }^{\circ} \mathrm{C}$ for $18 \mathrm{~h}$. The assay was repeated thrice. Wells containing MHB, $100 \mu \mathrm{L}$ of inoculum and DMSO to a final concentration of $2.5 \%$ served as negative control. The MIC of samples was detected after $18 \mathrm{~h}$ incubation at $37{ }^{\circ} \mathrm{C}$, following addition $(40 \mu \mathrm{L})$ of 0.2 $\mathrm{mg} / \mathrm{mL}$ of INT and incubation at $37{ }^{\circ} \mathrm{C}$ for $30 \mathrm{~min}$ as the lowest sample concentration that prevented the color change of the medium and exhibited complete inhibition of microbial growth [17]. The MBC was determined by adding $50 \mu \mathrm{L}$ aliquots of the preparations, which did not show any growth after incubation during MIC assays, to $150 \mu \mathrm{L}$ of MHB. These preparations were incubated at $37{ }^{\circ} \mathrm{C}$ for $48 \mathrm{~h}$. The $\mathrm{MBC}$ was regarded as the lowest concentration of samples, which did not produce a color change after addition of INT as mentioned above [20, 21].

\section{Results}

\section{Structural determination}

The chemical structures of compounds from the leaves of E. drupifera were elucidated using physical and NMR data and comparison with literature. The isolated compounds were identified as the mixture (1:3) of stigmasterol and $\beta$-sitosterol $(\mathbf{1}+2)$ [22]; euphol $\mathrm{C}_{30} \mathrm{H}_{50} \mathrm{O}$ (3; m.p. $113.8-114.5{ }^{\circ} \mathrm{C} ; m / z 426 ;[\alpha]_{\mathrm{D}}+31$ (c $\left.0.2, \mathrm{CH}_{2} \mathrm{Cl}_{2}\right)$ ) [23], sitosterol-O- $\beta$ - ${ }^{-}$-xylopyranoside $\mathrm{C}_{34} \mathrm{H}_{58} \mathrm{O}_{5}$ (4; m.p. $271-273{ }^{\circ} \mathrm{C} ; m / z$ 546; $[\alpha]_{\mathrm{D}}-50.1$ (c 0.9, DMSO- $\left.d_{6}\right)$ ) [24], 3,3',4'-tri-O-methylellagic acid $\mathrm{C}_{17} \mathrm{H}_{12} \mathrm{O}_{8}$ (5; m.p. $287-288.5{ }^{\circ} \mathrm{C}$; $\mathrm{m} / z$ 344) [25], the mixture (1:1) of afzelin and quercetin-3-O- $\beta-_{-}$-xylopyranoside $(6+7)[26,27] ; 3,3^{\prime}, 4^{\prime}$-tri- $O$-methylellagic acid $4-O-\beta^{-}{ }^{-}$ glucopyranoside $\mathrm{C}_{23} \mathrm{H}_{22} \mathrm{O}_{13}$ (8; m.p. $260-262{ }^{\circ} \mathrm{C} ; \mathrm{m} / z$ 506) [28] and ellagic acid-4-O- $\beta$-xylopyranoside- $3,3^{\prime}, 4^{\prime}-$ trimethyl ether $\mathrm{C}_{22} \mathrm{H}_{20} \mathrm{O}_{12}$ (9; m.p. $196.5-198.1{ }^{\circ} \mathrm{C} ; \mathrm{m} / z$ 476) [29] (Fig. 1). Extracts, fractions, compounds and the mixtures of two compounds identified in the leaves of $E$. drupifera were tested for their antimicrobial activities on a panel of Gram-negative and Gram-positive bacteria. The results are reported in Tables 1, 2, 3 and 4.

\section{Antibacterial activity}

Crude leaves extract (EDL), fractions EDLa-d and chloramphenicol were tested on a panel of 33 bacteria. The 
<smiles>CC[C@H](/C=C/[C@H](C)[C@H]1CCC2C3CC=C4C[C@@H](O)CC[C@]4(C)C3CC[C@]21C)C(C)C</smiles>

$1+2(1: 3)$

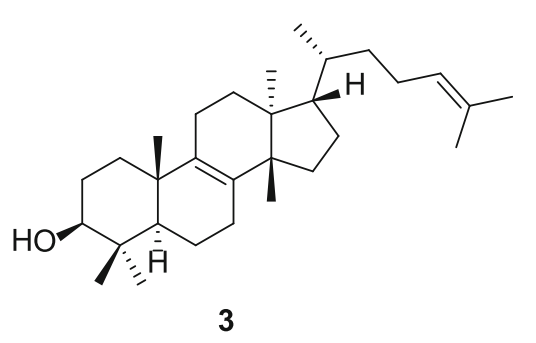

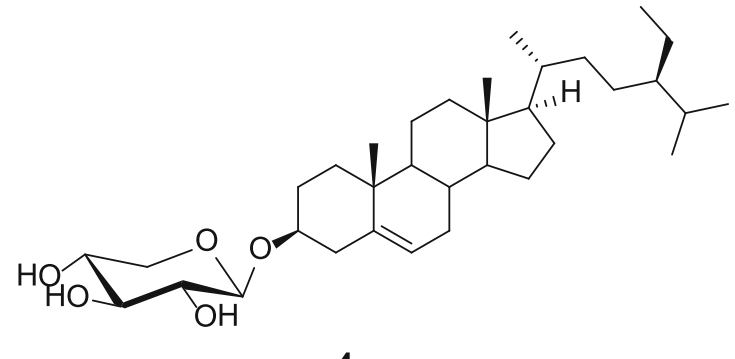

4<smiles>COc1cc2c(=O)oc3c(O)c(OC)cc4c(=O)oc(c1O)c2c34</smiles>

5<smiles>[R]Oc1c(-c2ccc(O)c(O)c2)oc2cc(O)cc(O)c2c1=O</smiles><smiles>COc1cc2c(=O)oc3c(OC)c(OC4OC(CO)C(O)[C@H](O)[C@H]4O)cc4c(=O)oc(c1OC)c2c34</smiles>

6+7 (1:1) R= Rhamnopyranosyl $\mathrm{R}^{1}=$ Xylopyranosyl

8<smiles></smiles>

Fig. 1 Chemical structures of compounds isolated from the leaves of Elaoephorbia drupifera. Mixture of stigmasterol and $\beta$-sitosterol (1+2); euphol (3); sitosterol-O- $\beta$ - ${ }_{-}$-xylopyranoside (4); 3,3',4'-tri-O-methylellagic acid (5); mixture of afzelin and quercetin-3-O- $\beta$ - ${ }^{-}$-xylopyranoside (6 + 7); 3,3', ''-tri-O-methylellagic acid 4-O- $\beta$-D-glucopyranoside (8); ellagic acid-4-O- $\beta$-xylopyranoside-3,3',4'-trimethyl ether (9)

results summarized in Table 1 reveal selective activities with MIC values ranged from 32 to $1024 \mu \mathrm{g} / \mathrm{mL}$ for EDL against 28/33 (84.9\%) tested bacteria as well as EDLc, EDLb, EDLa and EDLd respectively against 31/33 (93.9\%), 23/33 (69.7\%), 12/33 (33.4\%) and 1/33 (0.03\%) tested bacteria. MIC values below $1024 \mu \mathrm{g} / \mathrm{mL}$ were also recorded with chloramphenicol on 32/33 (97.0\%) tested bacteria. MBC values below1024 $\mu \mathrm{g} / \mathrm{mL}$ were obtained with EDLa-d as well as their mother extract EDL and RA on some of the tested bacterial strains. However, the recorded values were generally high. Table 2 reports the
MIC and MBC values of sub-fractions from EDLc (EDLc1-7) against a panel of 26 bacteria. It appears that MIC values ranged from 16 to $1024 \mu \mathrm{g} / \mathrm{mL}$ were obtained with EDLc3 and EDLc4 on all tested bacteria meanwhile other sub-fractions displayed selective activities. Their inhibitory effects were noted on $24 / 26$ (92.3\%), 22/26 (84.6\%), 13/26 (50.0\%), 10/26 (38.5\%) and $8 / 26(30.8 \%)$ tested bacteria. Further investigations of EDLc3 yielded five new sub-fractions (EDL31 to EDL35) with selective activities (Table 3). MIC values ranged from 8 to $1024 \mu \mathrm{g} / \mathrm{mL}$ on 23/24 (95.8\%) tested bacteria 
Table $1 \mathrm{MIC}$ and MBC (in $\mu \mathrm{g} / \mathrm{mL}$ ) of crude extract, fractions of E. drupifera leaves and chloramphenicol against the panel of 33 bacteria

\begin{tabular}{|c|c|c|c|c|c|c|}
\hline \multirow[t]{3}{*}{ Bacterial strains } & \multicolumn{6}{|c|}{ Tested samples and MICs MIC and MBC in parenthesis (in $\mu \mathrm{g} / \mathrm{mL}$ ) } \\
\hline & \multirow{2}{*}{$\begin{array}{l}\text { Crude extract } \\
\text { EDL }\end{array}$} & \multicolumn{4}{|l|}{ Fractions } & \multirow{2}{*}{$\begin{array}{l}\text { Reference drug } \\
\mathrm{CHL}\end{array}$} \\
\hline & & EDLa & EDLb & EDLC & EDLd & \\
\hline \multicolumn{7}{|l|}{ Escherichia coli } \\
\hline ATCC8739 & - & $64(1024)$ & $128(1024)$ & $64(1024)$ & - & $2(128)$ \\
\hline ATCC10536 & $256(-)$ & - & - & $128(1024)$ & - & $<2(64)$ \\
\hline AG100 & $1024(-)$ & $32(512)$ & - & $128(512)$ & - & $8(128)$ \\
\hline AG100A & $512(-)$ & $1024(-)$ & $1024(-)$ & $256(1024)$ & $512(-)$ & $<2(128)$ \\
\hline AG100A $A_{\text {TET }}$ & $1024(-)$ & $1024(-)$ & $1024(-)$ & - & - & $32(-)$ \\
\hline AG102 & $512(-)$ & $256(-)$ & $256(1024)$ & $256(-)$ & - & $64(-)$ \\
\hline MC4100 & $256(-)$ & - & - & $512(-)$ & - & $16(-)$ \\
\hline W3110 & $512(-)$ & $256(1024)$ & $128(1024)$ & $128(-)$ & - & $2(-)$ \\
\hline \multicolumn{7}{|c|}{ Pseudomonas aeruginosa } \\
\hline PA01 & - & $1024(-)$ & $1024(-)$ & $256(-)$ & - & $32(-)$ \\
\hline PA124 & - & $1024(-)$ & - & $128(-)$ & - & $128(-)$ \\
\hline \multicolumn{7}{|c|}{ Enterobacter aerogenes } \\
\hline ATCC13048 & - & - & - & $32(512)$ & - & $4(32)$ \\
\hline EA-CM64 & $512(-)$ & - & $512(1024)$ & $1024(-)$ & - & $256(-)$ \\
\hline EA3 & $1024(-)$ & - & $512(-)$ & $64(512)$ & - & $256(-)$ \\
\hline EA27 & $512(-)$ & - & - & $512(-)$ & - & $32(-)$ \\
\hline EA289 & $512(-)$ & $1024(-)$ & $512(-)$ & $128(1024)$ & - & $64(-)$ \\
\hline EA298 & $512(-)$ & - & $256(1024)$ & $32(128)$ & - & 128 \\
\hline \multicolumn{7}{|c|}{ Providencia stuartii } \\
\hline NEA16 & $512(-)$ & - & $256(512)$ & $256(-)$ & - & $32(256)$ \\
\hline ATCC29916 & $1024(-)$ & - & $256(1024)$ & $256(512)$ & - & $16(256)$ \\
\hline PS2636 & $512(-)$ & $512(-)$ & $256(-)$ & $64(512)$ & - & $16(256)$ \\
\hline PS299645 & $1024(-)$ & - & - & $64(128)$ & - & $64(-)$ \\
\hline \multicolumn{7}{|c|}{ Klebsiella pneumoniae } \\
\hline ATCC11296 & $512(-)$ & - & $1024(-)$ & $128(512)$ & - & $8(256)$ \\
\hline KP55 & $512(-)$ & $1024(-)$ & $256(1024)$ & $128(512)$ & - & $32(256)$ \\
\hline KP63 & $512(-)$ & $1024(-)$ & $1024(-)$ & $128(512)$ & - & $32(-)$ \\
\hline K24 & $512(-)$ & - & - & - & - & $64(256)$ \\
\hline $\mathrm{K} 2$ & $1024(-)$ & - & $512(1024)$ & $64(512)$ & - & $8(256)$ \\
\hline \multicolumn{7}{|c|}{ Enterobacter cloacae } \\
\hline BM47 & - & - & $256(-)$ & $1024(-)$ & - & $256(-)$ \\
\hline BM67 & $512(-)$ & - & $512(-)$ & $256(1024)$ & - & - \\
\hline BM94 & $512(-)$ & - & $1024(-)$ & $1024(-)$ & - & $128(-)$ \\
\hline \multicolumn{7}{|c|}{ Staphylococcus aureus } \\
\hline ATCC 25923 & $128(1024)$ & - & $1024(-)$ & $512(-)$ & - & $8(128)$ \\
\hline MRSA 3 & $1024(-)$ & - & - & $1024(-)$ & - & $16(-)$ \\
\hline MRSA 4 & $512(-)$ & - & - & $1024(-)$ & - & $64(-)$ \\
\hline MRSA 11 & $512(-)$ & - & $1024(-)$ & $512(-)$ & - & $32(-)$ \\
\hline MRSA 12 & $512(-)$ & - & 256 (1024) & $128(1024)$ & - & $16(256)$ \\
\hline
\end{tabular}

Tested samples were crude methanol extract of E. drupifera (EDL), hexane fraction (EDLa), dichloromethane fraction (EDLb), ethyl acetate fraction (EDLc), residual fraction (EDLC), and chloramphenicol (CHL); -: MIC or MBC values above $1024 \mu \mathrm{g} / \mathrm{mL}$ 
Table 2 MIC and MBC (in $\mu \mathrm{g} / \mathrm{mL}$ ) of sub-fractions of ethyl acetate fraction EDLc against the panel of 26 bacteria

\begin{tabular}{|c|c|c|c|c|c|c|c|}
\hline \multirow[t]{2}{*}{ Bacterial strains } & \multicolumn{7}{|c|}{ Sub-fractions, MIC and MBC in parenthesis (in $\mu \mathrm{g} / \mathrm{mL}$ ) } \\
\hline & EDLC1 & EDLC2 & EDLC3 & EDLC4 & EDLC5 & EDLc6 & EDLC7 \\
\hline \multicolumn{8}{|l|}{ Escherichia coli } \\
\hline ATCC8739 & $1024(-)$ & $512(-)$ & $64(-)$ & $512(-)$ & $512(-)$ & - & - \\
\hline ATCC 10536 & - & $1024(-)$ & $32(-)$ & $64(-)$ & $512(-)$ & $1024(-)$ & $1024(-)$ \\
\hline AG100A & - & $512(-)$ & $64(256)$ & $256(-)$ & - & - & - \\
\hline AG102 & - & $128(-)$ & $32(256)$ & $256(-)$ & $64(512)$ & $1024(-)$ & - \\
\hline W3110 & - & - & $16(-)$ & $64(-)$ & $512(-)$ & 1024 & - \\
\hline \multicolumn{8}{|c|}{ Pseudomonas aeruginosa } \\
\hline PA 01 & - & $128(-)$ & $64(-)$ & $512(-)$ & $1024(-)$ & $1024(-)$ & $1024(-)$ \\
\hline PA 124 & - & $1024(-)$ & $64(-)$ & $256(-)$ & $512(-)$ & $1024(-)$ & $1024(-)$ \\
\hline \multicolumn{8}{|c|}{ Enterobacter aerogenes } \\
\hline ATCC13048 & $512(-)$ & $256(-)$ & $128(256)$ & $512(-)$ & $1024(-)$ & - & $1024(-)$ \\
\hline EA-CM64 & - & $1024(-)$ & $64(256)$ & $512(-)$ & - & - & - \\
\hline EA289 & - & $128(-)$ & $32(-)$ & $64(-)$ & $512(-)$ & $1024(-)$ & $512(-)$ \\
\hline EA27 & $1024(-)$ & $1024(-)$ & $128(-)$ & $256(-)$ & $512(-)$ & - & $1024(-)$ \\
\hline \multicolumn{8}{|c|}{ Providencia stuartii } \\
\hline NEA16 & $1024(-)$ & $512(-)$ & $64(-)$ & $256(-)$ & - & - & - \\
\hline ATCC29916 & - & $256(-)$ & $64(-)$ & $256(-)$ & $512(-)$ & - & - \\
\hline PS2636 & $512(-)$ & $128(-)$ & $16(-)$ & $64(-)$ & $256(-)$ & $1024(-)$ & $512(-)$ \\
\hline PS299645 & $128(-)$ & $128(-)$ & $16(-)$ & $256(-)$ & $512(-)$ & $1024(-)$ & $512(-)$ \\
\hline \multicolumn{8}{|c|}{ Klebsiella pneumoniae } \\
\hline ATCC11296 & $1024(-)$ & $512(-)$ & $64(-)$ & $256(-)$ & $512(-)$ & - & - \\
\hline KP55 & - & $128(-)$ & $32(-)$ & $512(-)$ & $1024(-)$ & $512(-)$ & $1024(-)$ \\
\hline KP63 & - & $1024(-)$ & $64(-)$ & $512(-)$ & $512(-)$ & - & $1024(-)$ \\
\hline K2 & $256(-)$ & $64(-)$ & $16(-)$ & $64(-)$ & - & - & $512(-)$ \\
\hline \multicolumn{8}{|c|}{ Enterobacter cloacae } \\
\hline BM47 & - & $256(1024)$ & $64(256)$ & $256(-)$ & $512(-)$ & - & - \\
\hline BM67 & - & $512(-)$ & $64(256)$ & $256(-)$ & $1024(-)$ & - & - \\
\hline BM94 & - & $1024(-)$ & $32(256)$ & $64(-)$ & $1024(-)$ & - & $1024(-)$ \\
\hline \multicolumn{8}{|c|}{ Staphylococcus aureus } \\
\hline ATCC25923 & - & - & $64(512)$ & $512(-)$ & $1024(-)$ & - & - \\
\hline MRSA 3 & - & $1024(-)$ & $64(256)$ & $256(-)$ & $512(-)$ & - & - \\
\hline MRSA 4 & - & $256(-)$ & $32(256)$ & $256(-)$ & $512(-)$ & - & - \\
\hline MRSA 11 & - & $1024(-)$ & $16(-)$ & $128(-)$ & $256(-)$ & $1024(-)$ & $1024(-)$ \\
\hline
\end{tabular}

$-:$ MIC or MBC values above $1024 \mu \mathrm{g} / \mathrm{mL}$

for EDLc32, EDLc34 and EDLc35. It should be noted that MBC values noted with EDL31-EDL35 were generally above $1024 \mu \mathrm{g} / \mathrm{mL}$. A keen look of data from Tables 1 and 3 indicated that the ratio $\mathrm{MBC} / \mathrm{MIC}$ were generally above 4 . The antibacterial activities of the isolated compounds and the mixtures of two compounds are compiled in Table 4. Compound $\mathbf{5}$ as well as the mixture (1:1) of $\mathbf{6}$ and 7 inhibited the growth of all the 14 tested bacteria with MIC values ranged from 64 to
$256 \mu \mathrm{g} / \mathrm{mL}$. Compounds 3 and $\mathbf{8}$ inhibited respectively the growth of $1 / 14(7.1 \%)$ and $13 / 14$ (92.9\%) tested bacteria meanwhile $\mathbf{4}, \mathbf{9}$ as well as the mixture (1:3) of $\mathbf{1}$ and 2 were not active at up to $256 \mu \mathrm{g} / \mathrm{mL}$.

\section{Discussion}

In the present study, we identified 9 compounds amongst which were 4 terpenoids (1-4), 2 flavonoid glycosides (6 and 7), 3 ellagic acid derivatives (5, 8 and 9). 
Table 3 MIC and MBC (in $\mu \mathrm{g} / \mathrm{mL}$ ) activities of sub-fractions EDLC3 against the panel of 24 bacteria

\begin{tabular}{|c|c|c|c|c|c|}
\hline \multirow[t]{2}{*}{ Bacterial strains } & \multicolumn{5}{|c|}{ Sub-fractions, MIC and MBC in parenthesis (in $\mu \mathrm{g} / \mathrm{mL}$ ) } \\
\hline & EDLC31 & EDLC32 & EDLC33 & EDLC34 & EDLC35 \\
\hline \multicolumn{6}{|l|}{ Escherichia coli } \\
\hline ATCC8739 & $-(-)$ & $256(-)$ & $64(-)$ & $256(-)$ & $512(-)$ \\
\hline ATCC 10536 & $256(-)$ & $64(-)$ & $64(-)$ & $32(-)$ & $128(-)$ \\
\hline AG100A & $128(-)$ & $64(-)$ & $32(-)$ & $128(512)$ & $256(-)$ \\
\hline AG102 & $128(-)$ & $128(-)$ & $32(1024)$ & $256(1024)$ & $1024(1024)$ \\
\hline \multicolumn{6}{|c|}{ Pseudomonas aeruginosa } \\
\hline PA 01 & $256(-)$ & $32(-)$ & $256(1024)$ & $32(1024)$ & $64(-)$ \\
\hline PA 124 & - & - & - & - & - \\
\hline \multicolumn{6}{|c|}{ Enterobacter aerogenes } \\
\hline ATCC13048 & - & $256(512)$ & $512(-)$ & $128(-)$ & $256(512)$ \\
\hline EA-CM64 & - & $512(-)$ & $256(-)$ & $256(1024)$ & $256(-)$ \\
\hline EA289 & $128(-)$ & $32(-)$ & $64(-)$ & $32(-)$ & $64(-)$ \\
\hline EA27 & - & $128(-)$ & $256(-)$ & $512(-)$ & $256(-)$ \\
\hline \multicolumn{6}{|c|}{ Providencia stuartii } \\
\hline NEA16 & - & $32(-)$ & $8(-)$ & $32(-)$ & $128(1024)$ \\
\hline ATCC29916 & $512(-)$ & $64(-)$ & $512(-)$ & $64(-)$ & $128(-)$ \\
\hline PS2636 & $128(-)$ & $32(-)$ & $128(-)$ & $16(-)$ & $64(-)$ \\
\hline PS299645 & - & $128(-)$ & $512(-)$ & $1024(-)$ & $1024(-)$ \\
\hline \multicolumn{6}{|c|}{ Klebsiella pneumoniae } \\
\hline ATCC11296 & $512(-)$ & $256(-)$ & - & $64(-)$ & $128(-)$ \\
\hline KP63 & - & $32(512)$ & $64(1024)$ & $128(1024)$ & $256(-)$ \\
\hline K2 & $16(-)$ & $128(-)$ & $64(-)$ & $16(-)$ & $32(-)$ \\
\hline \multicolumn{6}{|c|}{ Enterobacter cloacae } \\
\hline $\mathrm{BM} 47$ & - & $256(-)$ & $1024(-)$ & $128(-)$ & $256(-)$ \\
\hline BM67 & - & $256(512)$ & $64(-)$ & $64(-)$ & $128(1024)$ \\
\hline BM94 & - & $64(-)$ & $256(-)$ & $1024(-)$ & $1024(-)$ \\
\hline \multicolumn{6}{|c|}{ Staphylococcus aureus } \\
\hline ATCC25923 & - & $256(-)$ & $256(-)$ & $128(-)$ & $256(-)$ \\
\hline MRSA 3 & - & $256(-)$ & 64 & $64(1024)$ & $128(1024)$ \\
\hline MRSA 4 & - & $256(-)$ & 256 & $64(-)$ & $128(-)$ \\
\hline MRSA 11 & $256(-)$ & $64(-)$ & 128 & 16 (1024) & 64 (1024) \\
\hline
\end{tabular}

$-:$ MIC or MBC values above $1024 \mu \mathrm{g} / \mathrm{mL}$

Among terpenoids were steroids (1 and $\mathbf{2}$ ), a steroid glucoside (4) and a triterpenoid (3). The isolation of compounds such as euphol (3), tirucallol, euphorbol, ingenol elaeophorbate, epitaraxerol, taraxerone, friedelin, lup20(29)-en-3-one or lupenone, lupeol, olean-12-ene-3one, olean-12-ene-3-ol, elaeophorbate in E. drupifera was previously reported $[14,15]$. However, in the present work, fewer compounds as well as other not previously isolated ones were isolated, probably due to the fact that the purification was guided by the antibacterial activity and hence all fractions and sub-fractions were not
Table 4 MIC (in $\mu \mathrm{g} / \mathrm{ml}$ ) of compounds isolated from of E. drupifera leaves against the panel of 14 bacteria

\begin{tabular}{|c|c|c|c|c|}
\hline \multirow[t]{2}{*}{ Bacterial strains } & \multicolumn{4}{|c|}{ Compounds and MIC (in $\mu \mathrm{g} / \mathrm{ml}$ ) } \\
\hline & 3 & 5 & $6+7$ & 8 \\
\hline \multicolumn{5}{|l|}{ Escherichia coli } \\
\hline ATCC8739 & - & 128 & 64 & 256 \\
\hline ATCC10536 & - & 128 & 128 & 256 \\
\hline AG102 & - & 128 & 64 & 64 \\
\hline \multicolumn{5}{|c|}{ Pseudomonas aeruginosa } \\
\hline PA 01 & - & 128 & 128 & - \\
\hline PA 124 & 256 & 128 & 128 & 64 \\
\hline \multicolumn{5}{|c|}{ Enterobacter aerogenes } \\
\hline ATCC13048 & - & 128 & 64 & 128 \\
\hline EA-CM64 & - & 128 & 128 & 128 \\
\hline \multicolumn{5}{|c|}{ Providencia stuartii } \\
\hline ATCC29916 & - & 128 & 128 & 256 \\
\hline PS2636 & - & 64 & 64 & 256 \\
\hline \multicolumn{5}{|c|}{ Klebsiella pneumoniae } \\
\hline ATCC11296 & - & 128 & 128 & 256 \\
\hline KP55 & - & 128 & 128 & 128 \\
\hline \multicolumn{5}{|c|}{ Staphylococcus aureus } \\
\hline ATCC 25923 & - & 128 & 64 & 64 \\
\hline MRSA3 & - & 256 & 128 & 64 \\
\hline MRSA4 & - & 256 & 128 & 256 \\
\hline
\end{tabular}

Tested compounds were a mixture (1:3) of stigmasterol and $\beta$-sitosterol $(\mathbf{1}+\mathbf{2})$; euphol (3); sitosterol-O- $\beta$ - ${ }^{-}$-xylopyranoside (4); 3,3',4'-tri-O-methylellagic acid (5); mixture 1:1 of afzelin and quercetin-3-O- $\beta$-D-xylopyranoside $(\mathbf{6}+\mathbf{7}) ; 3,3^{\prime}, 4^{\prime}$-tri-Omethylellagic acid 4-O- $\beta$-o-glucopyranoside (8); ellagic acid-4-O- $\beta$-xylopyranoside$3,3^{\prime}, 4^{\prime}$-trimethyl ether (9). No MBC was detected at $256 \mu \mathrm{g} / \mathrm{mL}$ for all compounds; MIC values were $>256 \mu \mathrm{g} / \mathrm{mL}$ for $\mathbf{1}+\mathbf{2}, \mathbf{4}$ and $\mathbf{9}$

explored. According to established criteria, the antibacterial activity of a plant extract is considered to be significant when MIC is below $100 \mu \mathrm{g} / \mathrm{mL}$, moderate when $100 \mu \mathrm{g} / \mathrm{mL}<\mathrm{MIC}<625 \mu \mathrm{g} / \mathrm{mL}$ or low when $\mathrm{MIC}>100 \mu \mathrm{g} / \mathrm{mL}[30,31]$. Therefore, the antibacterial activity of the leaves extract (EDL) of E. drupifera could be considered as moderate, as MIC values below $625 \mu \mathrm{g} / \mathrm{mL}$ were obtained on the majority of the tested bacteria (Table 1). However, fractionation of EDL afforded more active samples, and the antibacterial effects of EDLc, EDLc3, EDLc4, EDLc32 to EDLc35 could be considered important. In fact, the lowest MIC value of $32 \mu \mathrm{g} / \mathrm{mL}$ was obtained with fractions EDLa against Escherichia coli AG100, EDLc against Enterobacer aerogenes ATCC13048and EA298. For sub-fractions obtained from EDLc, the lowest MIC value of $16 \mu \mathrm{g} / \mathrm{mL}$ was noted with EDLc3 against Staphylococcus aureus MRSA11. A corresponding value of $8 \mu \mathrm{g} / \mathrm{mL}$ against Providencia stuartii NAE16 was recorded with EDLc33 obtained from further fractionation of 
EDLc3. These data highlight the increase of activity with consecutive fractionation of extracts and also demonstrate the good antibacterial potential of $E$. drupifera. Imporantly, the MIC values obtained with the best sub-fractions EDLc3 against Pseudomonas aeruginosa PA124, E. aerogenes EA289, Providencia stuartii PS29964, Enterobacter cloacae BM47, BM67, BM94, S. aureus MRSA4 (Table 2) and EDLc33 against P. stuartii NAE16 (Table 2) were lower than those of chormaphenicol. It should also be highlighted that EDLc3 had MIC values below $100 \mu \mathrm{g} / \mathrm{mL}$ against all the 26 tested bacteria (Table 2). The ratio $\mathrm{MBC} / \mathrm{MIC}$ obtained were generally above 4 , indicating that the studied extracts as well as the active fractions mostly exerted bacteriostatic effects [32-34]. Also, MIC and MBC values of the reference drug chloramphenicol were also very high $(>64 \mu \mathrm{g} / \mathrm{mL})$ on several pathogens, confirming that most of the bacterial strains used were MDR phenotypes. The activity of compounds is significant when $\mathrm{MIC}<10 \mu \mathrm{g} / \mathrm{mL}$, moderate when $10<\mathrm{MIC}<100 \mu \mathrm{g} / \mathrm{mL}$ and low when MIC $>100 \mu \mathrm{g} / \mathrm{mL}[30,31]$. On this basis, none of the compound or mixtures displayed significant antibacterial activity. Also the lowest MIC value of $64 \mu \mathrm{g} / \mathrm{mL}$ obtained with compound $\mathbf{5}$ was much more higher than the corresponding values for the most active fraction EDLc3 where it were isolated. This suggests that constituents of this fraction may exert synergistic effects. This also indicates that combating the tested bacteria with fractions and mostly EDLc3 could be more efficient than with isolated compounds. When regarding the structure-activity relationship, it appears that terpenoids were poorly or not active against the tested bacteria. This result is not suprising, as terpenoids are known to generally have poor antibacterial activity [30]. Amongst the three ellagic acid derivatives, compound $\mathbf{5}$ and the glucoside $\mathbf{8}$ had antibacterial activities contrary to 9 . Hence, it may be deduced that the substitution of a glucosyl- (8) group by the xylopyranosylgroup (9) cancels the antibacterial effect of the ellagic acid derivative 9.

To the best of our knowledge, the identification of the antibacterial constituents of E. drupifera is being reported for the first time. However, the antibacterial activity of the leave of extract of the plant was reported on Staphylococcus aureus and Streptococcus pyogenes [9]. The present study therefore provides more information on the antibacterial potential of $E$. drupifera and identified the bioactive components of plant. Also, compound 5 was previously reported against a panel of sensitive Gram-positive and Gram-negative bacteria, with MIC values ranged from 9.76 to $156.25 \mu \mathrm{g} / \mathrm{mL}$ [18]. Data obtained in the present study (MIC ranged from 64 to $256 \mu \mathrm{g} / \mathrm{mL}$ ) are in consistence with previous and also confirm the activity of compound $\mathbf{5}$ against MDR bacteria.

\section{Conclusions}

The results of the present investigation are very interesting, taking in account the medical importance of the studied microorganisms. The most active fraction of the plant, identified as EDLc3 displayed significant antibacterial activity on a panel of 26 tested bacteria including both sensitive and MDR Gram-negative and Gram-positive bacteria. This fraction could therefore be useful in the management of bacterial infection including MDR phenotypes. The bioactive constituents of the plant include $3,3^{\prime}, 4^{\prime}$-tri- $O$-methylelleargic acid, 3-O-rhamnopyranosyl kaempherol, 3-O-rhamnopyranosyl quercetin and $3,3^{\prime}, 4^{\prime}$-tri- $O$-méthylelleargic acid

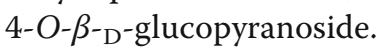

\section{Additional file}

Additional file 1: Table S1. Bacterial strains used and their features. (DOCX 2625 kb)

\begin{abstract}
Abbreviations
1: Stigmasterol; 2: $\beta$-sitosterol; 3: Euphol; 4: Sitosterol-O- $\beta$ - ${ }^{-}$-xylopyranoside; 5: 3,3',4'-tri-O-methylellagic acid; 6: Afzelin; 7: Quercetin-3-O- $\beta_{-}{ }^{-}$ xylopyranoside; 8 : 3,3', $4^{\prime}$-tri-O-methylellagic acid $4-O-\beta$-D-glucopyranoside; 9: Ellagic acid-4-O- $\beta$-xylopyranoside-3,3',4'-trimethyl ether; ATCC: American Type Culture Collection; CFU: Colony forming unit; DMSO: Dimethyl sulfoxide; E. aerogenes: Enterobacter aerogenes; E. cloacae: Enterobacter cloacae; E. coli: Escherichia coli; EDL: Methanol extract from the leaves of Elaeophorbia drupifera; EDLa-d: Fractionsfrom EDL; EDLc1-7: Sub-fractions from EDLC; $\mathrm{H}_{2} \mathrm{SO}_{4}$ : Sulphuric acid; HNC: National Herbarium of Cameroon; INT: $p$-iodonitrotetrazolium chloride $\geq 97 \%$ (INT, Sigma-Aldrich).; $K$. pneumoniae: Klebsiella pneumoniae; MBC: Minimal bactericidal concentration; MDR: Multidrug resistant; MDR: Multi-drug resistant; MHB: Mueller Hinton Broth; MIC: Minimal inhibitory concentration; P. aeruginosa: Pseudomonas aeruginosa; P. stuartii: Providencia stuartii; RA: Reference antibiotic; TLC: Thin layer chromatography
\end{abstract}

\section{Acknowledgements}

Authors are thankful to the Cameroon National Herbarium (Yaounde) for the plant identification. Authors are also thankful to UMR-MD1 (Mediterranean University, Marseille, France) for providing some clinical bacteria. Author would like to thank Department of Chemistry, Anadolu University for providing NMR spectra (project number: 1306F110).

\section{Funding}

No funding.

\section{Availability of data and materials}

The datasets supporting the conclusions of this article are presented in this main paper. Plant materials used in this study have been identified at the

Cameroon National Herbarium where voucher specimens are deposited.

\section{Authors' contributions}

IKV and BKN carried out the study; VK designed the experiments and wrote the manuscript; IC and PLS contributed to structural elucidation; VK and VPB supervised the work; VK provided the bacterial strains; all authors read and approved the final manuscript.

\section{Competing interests}

The authors declare that they have no competing interests.

Consent for publication

Not applicable in this section. 


\section{Ethics approval and consent to participate}

Not applicable in this section.

\section{Author details}

'Department of Biochemistry, Faculty of Science, University of Dschang, P.O. Box 67, Dschang, Cameroon. ${ }^{2}$ Department of Chemistry, Faculty of Science, University of Dschang, Dschang, Cameroon. ${ }^{3}$ Department of Pharmaceutical Sciences, CCS, Universidade Federal de Santa Catarina, Florianópolis 88040-900, Santa Catarina, Brazil. ${ }^{4}$ Department of Chemistry, Faculty of Science, Anadolu University, Eskişehir, Turkey. ${ }^{5}$ Department of Biochemistry, Faculty of Science, University of Yaounde I, Yaoundé, Cameroon.

Received: 26 August 2016 Accepted: 2 December 2016

Published online: 07 January 2017

\section{References}

1. Rice LB. Unmet medical needs in antibacterial therapy. Biochem Pharmacol. 2006;71:991-5.

2. Ashraf A, Sarfraz RA, Rashid MA, Shahid M. Antioxidant, antimicrobial, antitumor, and cytotoxic activities of an important medicinal plant (Euphorbia royleana)from Pakistan. J Food Drug Anal. 2015;13:109-15.

3. Ashraf A, Sarfraz RA, Anwar F, Shahid SA, Alkharfy KM. Chemicalcomposition and biological activities of leaves of Ziziphus mauritiana L. native toPakistan. Pak J Bot. 2015:47:367-76.

4. Djeussi DE, Sandjo LP, Noumedem JA, Omosa LK BTN, Kuete V. Antibacterial activities of the methanol extracts and compounds from Erythrina sigmoidea against Gram-negative multi-drug resistant phenotypes. BMC Complement Altern Med. 2015:15:453.

5. Voukeng Kl, Kuete V, Dzoyem JP, Fankam AG, Noumedem KJA, Kuiate JR, Pages JM. Antibacterial and antibiotic-potentiation activities of the methanol extract of some Cameroonian spices against Gram-negative multi-drug resistant phenotypes. BMC Res Notes. 2012;5:299.

6. Voukeng Kl, Beng PV, Kuete V. Antibacterial activity of six medicinal Cameroonian plants against Gram-positive and Gram-negative multidrug resistant phenotypes. BMC Complement Altern Med. 2016:16:388.

7. Fankam AG, Kuiate JR, Kuete V. Antibacterial and antibiotic resistance modifying activity of the extracts from Allanblackia gabonensis, Combretum molle and Gladiolus quartinianus against Gram-negative bacteria including multi-drug resistant phenotypes. BMC Complement Altern Med. 2015;15:206

8. Tankeo SB, Damen F, Sandjo LP, Celik I, Tane P, Kuete V. Antibacterial activities of the methanol extracts, fractions and compounds from Harungana madagascariensis Lam. ex Poir. (Hypericaceae). J Ethnopharmacol. 2016;190:100-5.

9. Pesewu GA, Cutler RR, Humber DP. Antibacterial activity of plants used in traditional medicines of Ghana with particular reference to MRSA. J Ethnopharmacol. 2008;116:102-11.

10. Eno A, Azah N. Effect of ethanolic extract from Elaeophorbia drupifera leaves on the gastrointestinal smooth muscle of the rabbit. Nig J Physiol Sci. 2004;19:60-8.

11. Ayisi NK, Nyadedzor C. Comparative in vitro effects of AZT and extract of Ocimum gratissimum, Ficus polita, Clausena anisata, Alchornea cordifolia, and Elaeophorbia drupifera against HIV-1 and HIV-2 infections. Antivir Res. 2003;58:25-33.

12. Eno $A E$, Owo Ol. Cardiovascular effects of an extract from the roots of a shrub Elaeophorbia drupifera. Phytother Res. 1999;13:549-54.

13. Kuete V, Voukeng Kl, Tsobou R, Mbaveng TA, Wiench B, Beng PV, Efferth T. Cytotoxicity of Elaoephorbia drupifera and other Cameroonian medicinal plants against drug sensitive and multidrug resistant cancer cells. BMC Complement Altern Med. 2013;13:250.

14. Kinghorn A, Evans F. Occurrence of ingenol in Elaeophorbia species. Planta Med. 1974:26:150-4

15. Ahiahonu PW, Goodenowe DB. Triterpenoids from leaves of Elaeophorbia drupifera. Fitoterapia. 2007;78:337-41

16. Kuete $V$, Metuno R, Ngameni B, Tsafack AM, Ngandeu F, Fotso GW, Bezabih M, Etoa FX, Ngadjui BT, Abegaz BM, et al. Antimicrobial activity of the methanolic extracts and compounds from Treculia obovoidea (Moraceae). J Ethnopharmacol. 2007:112:531-6.

17. Eloff JN. A sensitive and quick microplate method to determine the minimal inhibitory concentration of plant extracts for bacteria. Planta Med. 1998:64:711-3.
18. Kuete V, Wabo GF, Ngameni B, Mbaveng AT, Metuno R, Etoa FX, Ngadjui BT, Beng VP, Meyer JJ, Lall N. Antimicrobial activity of the methanolic extract, fractions and compounds from the stem bark of Irvingia gabonensis (Ixonanthaceae). J Ethnopharmacol. 2007;114:54-60.

19. Djeussi DE, Noumedem JA, Seukep JA, Fankam AG, Voukeng IK, Tankeo SB, Nkuete AH, Kuete $V$. Antibacterial activities of selected edible plants extracts against multidrug-resistant Gram-negative bacteria. BMC Complement Altern Med. 2013;13:164

20. Kuete V, Nana F, Ngameni B, Mbaveng AT, Keumedjio F, Ngadjui BT. Antimicrobial activity of the crude extract, fractions and compounds from stem bark of Ficus ovata (Moraceae). J Ethnopharmacol. 2009:124:556-61.

21. Kuete V, Wansi JD, Mbaveng AT, Kana Sop MM, Tadjong AT, Beng VP, Etoa FX, Wandji J, Meyer JJM, Lall N. Antimicrobial activity of the methanolic extract and compounds from Teclea afzelii (Rutaceae). S Afr J Bot. 2008;74:572-6.

22. De-Eknamkul W. Potduang B. Biosynthesis of beta-sitosterol and stigmasterol in Croton sublyratus proceeds via a mixed origin of isoprene units. Phytochemistry. 2003;62(3):389-98.

23. Gewali M, Hattori M, Tezuka Y, Kikuchi T, Namba T. Constituents of the latex of Euphorbia antiquorum. Phytochemistry. 1990;29:1625-8.

24. Alam S, Ali M, Alam P, Shuaib M. Phytochemical investigation of the seeds of Butea monosperma. Chem Nat Comp. 2010;46:44-8.

25. Abd El Azim M, El-Gerby MA, Awad A. Anti-Tumor, antioxidant and antimicrobial and the phenolic constituents of clove flower buds (Syzygium aromaticum). J Microbial BiochemTechnol. 2014;007:S8.

26. Lee SY, So YJ, Shin MS, Cho JY, Lee J. Antibacterial effects of afzelin isolated from Cornus macrophylla on Pseudomonas aeruginosa, a leading cause of illness in immunocompromised individuals. Molecules. 2014;19:3173-80.

27. Zhu Y, Liu Y, Zhan Y, Liu L, Xu Y, Xu T, Liu T. Preparative isolation and purification of five flavonoid glycosides and one benzophenone galloyl glycoside from Psidium guajava by high-speed counter-current chromatography (HSCCC). Molecules. 2013;18:15648-61.

28. Ye G, Peng H, Fan M, Huang C. Ellagic acid derivatives from the stem bark of Dipentodon sinicus. Chem Nat Comp. 2007:43:125-7.

29. Sinha A, Taylor WH, Khan $\|_{1}$, McDaniel ST, Esko JD. Glycoside primers of Psittacanthus cucullaris. J Nat Prod. 1999:62:1036-8.

30. Kuete V. Potential of Cameroonian plants and derived products against microbial infections: a review. Planta Med. 2010;76:1479-91.

31. Kuete V, Efferth T. Cameroonian medicinal plants: pharmacology and derived natural products. Front Pharmacol. 2010;1:123.

32. Mims C, Playfair J, Roitt I, Wakelin D, Williams R. Antimicrobials and chemotherapy. Med Microbiol Rev. 1993;35:1-34. In: Mims CA, et al Eds.

33. Mbaveng AT, Kuete $V$, Mapunya BM, Beng VP, Nkengfack AE, Meyer JJ, Lall N. Evaluation of four Cameroonian medicinal plants for anticancer, antigonorrheal and antireverse transcriptase activities. Environ Toxicol Pharmacol. 2011;32:162-7

34. Mbaveng AT, Ngameni B, Kuete V, Simo IK, Ambassa P, Roy R, Bezabih M, Etoa FX, Ngadjui BT, Abegaz BM, et al. Antimicrobial activity of the crude extracts and five flavonoids from the twigs of Dorstenia barteri (Moraceae). J Ethnopharmacol. 2008;116:483-9.

\section{Submit your next manuscript to BioMed Central and we will help you at every step:}

- We accept pre-submission inquiries

- Our selector tool helps you to find the most relevant journal

- We provide round the clock customer support

- Convenient online submission

- Thorough peer review

- Inclusion in PubMed and all major indexing services

- Maximum visibility for your research

Submit your manuscript at www.biomedcentral.com/submit
C) Biomed Central 